\title{
RESEARCH
}

Open Access

\section{Evaluation of meaningful change in bowel movement frequency for patients with carcinoid syndrome}

Stacie Hudgens ${ }^{1 *}\left(\mathbb{D}\right.$, John Ramage ${ }^{2}$, Matthew Kulke ${ }^{3}$, Emily Bergsland ${ }^{4}$, Lowell Anthony ${ }^{5}$, Martyn Caplin ${ }^{6}$, Kjell Öberg ${ }^{7}$, Marianne Pavel ${ }^{8}$, Jonathon Gable ${ }^{1}$, Phillip Banks ${ }^{9}$, Qi Melissa Yang ${ }^{9}$ and Pablo Lapuerta ${ }^{9}$

\begin{abstract}
Background: Carcinoid syndrome is associated with a reduced quality of life that can be attributed to symptoms such as diarrhea and fatigue as well as social and financial issues. This study was conducted to psychometrically assess meaningful change in bowel movement frequency among carcinoid syndrome patients using data from the TELESTAR clinical study.

Methods: An anchor-based approach for deriving meaningful change thresholds consisted of mapping change from baseline bowel movement frequency to other patient-reported assessments of change. These included the European Organization for Research and Treatment of Cancer (EORTC) Quality of Life Questionnaire - Core Questionnaire (QLQ-C30) Diarrhea Symptom responders, the EORTC Gastrointestinal NET questionnaire (GI.NET21) Gl Symptom responders, and reported adequate relief at Week 12 ( $\geq 10$-point score decrease from Day 1 to Week 12). Parameters included within-group mean change from baseline to Week 12, t-tests of the change (Wilcoxon rank sum for adequate relief), and effect size.

Results: There were 135 carcinoid syndrome patients with a mean baseline frequency of 5.7 bowel movements a day. A distribution-based method yielded meaningful change estimates of 0.62 bowel movements a day for overall frequency and 0.83 bowel movements a day at Week 12. Anchor-based analysis indicated a large effect size among patients who reported adequate relief at Week $12(-1.58 ; n=18 ; P=0.014)$, the QLQ-C30 Diarrhea domain responders $(-1.24 ; n=40 ; P<0.001)$, and the GI.NET21 GI Symptoms Domain responders $(-1.49 ; n=25 ; P=0.005)$. Exit interview data for meaningful change yielded effect size estimates of -1.57 for overall change during the Double-blind Treatment Period and - 1.97 for change between Baseline and Week 12.

Conclusions: Meaningful change derivation is critical to interpret patient outcomes for evaluating treatment efficacy. In this study, carcinoid syndrome patients experienced clinically meaningful reductions in bowel movement frequency of $\geq 30 \%$ over 12 weeks with telotristat ethyl treatment.
\end{abstract}

Trial registration: NCT01677910.

Keywords: Carcinoid syndrome, Bowel movement frequency, Meaningful change

\footnotetext{
*Correspondence: stacie.hudgens@clinoutsolutions.com

${ }^{1}$ CEO \& Strategic Lead, Quantitative Science, Clinical Outcomes Solutions,

1790 E. River Rd, Suite 205, Tucson, AZ 85718, USA

Full list of author information is available at the end of the article
} 


\section{Introduction}

Carcinoid tumors are well-differentiated neuroendocrine tumors (NETs) that originate in neuroendocrine cells [1]. Advanced disease is associated with a reduced healthrelated quality of life (HRQoL) related to diarrhea, fatigue, and flushing symptoms, besides family, social, and financial issues [2-5]. These NETs usually occur in the small intestine and represent about $0.5 \%$ of all newly diagnosed malignancies with an annual incidence of approximately 2 per 100,000 persons $[6,7]$. The overall 5 -year survival rate is $67.2 \%$ [6]. Carcinoid syndrome (CS) is caused by bioactive compounds released into the circulation, occurring in almost $20 \%$ of NETs patients [8]. Large amounts of serotonin (5-hydroxytryptamine [5-HT]) release is believed to cause diarrhea, endocardial and mesenteric fibrosis [9-11].

The TELESTAR study was conducted to psychometrically assess meaningful change in bowel movement (BM) frequency and evaluate the efficacy and safety of telotristat ethyl in CS patients with diarrhea not adequately controlled by somatostatin analogs (SSA) (TELESTAR study; NCT01677910). The primary objective of TELESTAR was to confirm that at least 1 or more doses of telotristat ethyl was effective in reducing the number of daily BMs from baseline averaged over the 12-week double-blind period (Treatment Period) [12].

\section{Methods}

\section{Study design}

The design and results of TELESTAR have been previously described [13]. Briefly, patients entered a Screening/ Run-in Period of 3- or 4-weeks to establish Baseline symptoms. They were then randomly assigned (1:1:1) on Day 1 to receive one of two dose levels of telotristat ethyl (250 or $500 \mathrm{mg}$ ) or placebo thrice daily for 12 weeks. All patients remained on their baseline dose of SSA therapy during the Treatment Period. Subsequently, they participated in a 36-week Open-label Extension Period when everyone received $500 \mathrm{mg}$ of the active study drug thrice daily. This study received Institutional Review Board approval.

The focus is on the primary endpoint, change from Baseline in BM frequency during the Double-blind Treatment Period. The intent-to-treat (ITT) analysis population included all randomized patients. All analyses populations were derived from the ITT dataset. All patients participating in the patient interview substudy after the Double-blind Treatment Period were included in the patient interview subpopulation (ISP).

\section{Study instruments}

Evaluation of meaningful change in BM frequency required the inclusion of other supportive clinical outcomes assessments: a Yes/No question about CS gastrointestinal symptom relief; European Organization for Research and
Treatment of Cancer (EORTC) Quality of Life Questionnaire - Core Questionnaire (QLQ-C30) and EORTC Gastrointestinal NET questionnaire (GI.NET21) scales; and patient exit interview responses.

\section{Number of daily BMs}

Patients electronically recorded the number of daily BMs. The average BM number was mapped to individual analyses using the following criteria: difference between average Baseline BM frequency and the overall average BM frequency; and the difference between average Baseline BM frequency and average BM frequency at Week 12 .

\section{EORTC QLQ-C30 and GI.NET21}

The QLQ-C30 contained 30 questions incorporated into 5 functional domains (Physical, Role, Cognitive, Emotional, and Social), 9 symptom scales (Fatigue, Pain, Nausea and Vomiting, Dyspnea, Insomnia, Appetite Loss, Constipation, Diarrhea, and Financial Difficulties), and a single global HRQoL/Global Health Status score [14].

The GI.NET21 module contained 21 questions: 4 single-item assessments about muscle and/or bone pain, body image, information, and sexual functioning, plus 17 items organized into 5 scales: Endocrine Symptoms (3 items), GI Symptoms (5 items), Treatment-related Symptoms (3 items), Social Functioning (3 items) and Disease-related Worries (3 items) [15].

\section{Exit interviews}

English- and German-speaking patients were invited to participate in the exit interview study as prespecified in the TELESTAR protocol [16]. All participants consented to the interview procedure to be conducted within 2 weeks after they completed the 12-week Double-blind Treatment Period or early termination. Patients were categorized based on reported satisfaction with improvement over the course of treatment ("very satisfied"; "somewhat satisfied"; "neither satisfied nor dissatisfied"; "somewhat dissatisfied"; or "very dissatisfied"). Patients were also categorized according to perception of BM frequency reduction ("a great deal better"; "much better"; "a little better"; "the same"; "a little worse"; "much worse"; or "a great deal worse").

\section{Analytic methods}

Analyses focused on the derivation and evaluation of thresholds to interpret meaningful change and responsiveness in BM frequency. All patients were included irrespective of receiving treatment $(n=90)$ or placebo $(n=45)$. Analyses was conducted using SAS Version 9.3 or higher (SAS Institute, Cary, NC, USA) [17].

Meaningful change on a patient-centered endpoint referred to the smallest difference in scores in the domain 
of interest (e.g., symptom or functional score), which patients perceived as beneficial. This could then be used further to discriminate between treatment groups and develop a thorough understanding of the HRQOL impact of BM frequency reduction [18, 19].

Change in BM frequency from Baseline was used to develop two individual distribution-based estimates: (1) overall change from Baseline, defined as the difference between average BM frequency during the Run-In Period and average BM frequency during the DoubleBlind Treatment Period; and (2) change from Baseline at Week 12, defined as the difference between average BM frequency during the Run-In Period and 7-day average BM frequency at Week 12. Distribution-based thresholds were derived for both estimates independently using the $1 / 2$ standard deviation (SD) rule [18], which is $1 / 2$ the SD of both estimates.

The anchor-based approach to derive meaningful change thresholds consisted of mapping change from Baseline in BM frequency to other patient reported assessments of change. The relationship between BM frequency and each continuous patient-reported outcome (PRO) anchor was evaluated prior to inclusion in the anchor based analysis using correlational analyses. The criterion threshold value for determining if the anchor was correlated with the outcome is a correlation coefficient $>0.30$ at Baseline, Week 12, or change from Baseline [18]. Anchor-based thresholds were developed by calculating mean change and the associated effect size (ES) statistic for each anchor-based. The ES was calculated from the difference between average score in BM frequency over 12 weeks and average Baseline BM frequency, this difference being divided by the SD of average Baseline BM frequency. A negative ES represented BM frequency reduction compared to Baseline [19, 20]. An additional analysis was conducted, where ES was calculated as the mean score in average BM frequency at Week 12 minus the average Baseline BM frequency divided by the SD of the group average Baseline BM frequency. For both analyses, a single value (or range of values for interpreting change in BM frequency) was developed for the full ITT population by selecting the mean improvement for each analytic group. These thresholds could be applied to stratify patients within each treatment arm by mean change in BM frequency relative to the identified threshold. Negative values of ES indicated reductions from Baseline in BM frequency.

Responsiveness refers to the ability of an assessment to detect change where it exists [18]. To assess responsiveness, patients were defined as improved or worsened based on meaningful change in prespecified categorical endpoints. An absolute improvement of 10 points from Day 1 to Week 12 defined improvement in each of the EORTC domains [20]. The analysis of covariance
(ANCOVA) procedure was used to calculate the $P$-value and adjusted for age, sex, and race. The within-group level of change in individual scores was expressed as a standardized effect size (SES), calculated as the mean change score between Baseline and Week 12, and divided by the SD of the pooled population at Baseline. Based on Cohen's recommendations, the following values represent the magnitudes of responsiveness: small change $(S E S=0.20)$, moderate change $(S E S=0.50$ ), and large change $(\mathrm{SES}=0.80)$ [21]. Statistically significant differences in change in scores between groups were tested through an ANCOVA model adjusting for age, sex, and race.

To demonstrate the application of MCT in responsiveness evaluation, unblinded cumulative distribution function (CDF) curves are presented, calculated as the cumulative percentage of patients achieving various threshold levels of change from Baseline in either the overall average daily BMs or the average BMs during Week 12 by treatment arm.

\section{Results}

A total of 135 patients were included in the ITT population at baseline (Run-in Period), Day 1, and 120 patients were included at Week 12. Baseline characteristics were generally similar across all treatment groups [13]. The numbers of patients with available PRO data at Week 12 by parameter were: $n=108(90.0 \%)$ average daily BM; $n=114(95.0 \%)$ adequate relief; $n=113(94.2 \%)$ EORTC QLQ-C30; and n = 113 (94.2\%) GI.NET21 data.

\section{Interpretation of scores: meaningful change Relationship between potential PRO anchors and BM frequency}

Only the QLQ-C30 Diarrhea symptom score yielded a correlation coefficient with average BM frequency exceeding the acceptability threshold of $>0.30$ at Week 12 $($ Pearson $=0.42$; Spearman $=0.45)$. Correlation was at least equal to the acceptability threshold for two items: (1) between change from baseline in the QLQ-C30 Diarrhea symptom score at Week 12 and in overall BM frequency $($ Pearson $=0.38$; Spearman $=0.31) ;(2)$ and a correlation between change from baseline in GI.NET21 GI Symptoms domain score at Week 12 and change from baseline in overall BM frequency (Pearson $=0.30$; Spearman $=0.30$ ).

\section{Anchor-based analysis of meaningful overall change from baseline in BM frequency}

An anchor-based analysis of thresholds for meaningful overall change in BM frequency produced a large ES (defined as $>0.80$ ) among ITT patients reporting adequate relief at Week $12(-1.20)$, EORTC QLQ-C30 Diarrhea Symptom scale $(-0.83)$ and EORTC GI.NET21 
GI Symptoms scale $(-1.25)$ responders. Wilcoxon Rank Sum test analysis indicated a significant difference in adequate relief groups $(P$-value $=0.011)$, EORTC QLQ-C30 Diarrhea Symptoms domain $(P$-value $=0.019)$, and EORTC GI.NET21 GI Symptoms domain responders and non-responders $(P$-value $<0.001)$ (Table 1$)$.

Large ES estimates were observed among ISP patients whose perception of change was "a great deal better" ($1.57)$ and "much better" $(-1.52)$, patients who reported being "very satisfied" $(-1.30)$ and "somewhat dissatisfied" $(-1.24)$ with symptom relief, the collapsed improvement category for carcinoid symptom relief $(-0.93)$, and those that considered their perception of change in BM frequency to be clinically meaningful $(-0.99)$. Kruskal-Wallis Exact test indicated a significant difference between the perceptions of change in $\mathrm{BMs}$ response groups $(P$-value $=$ 0.005) (Fig. 1).

Analysis of meaningful change in BM frequency at week 12 Anchor-based analysis indicated a large ES among ITT patients who reported adequate relief at Week 12 (1.58), responders on the EORTC QLQ-C30 Diarrhea domain (-1.24), and the EORTC GI.NET21 GI Symptoms Domain (-1.49) (Table 1). Wilcoxon Rank Sum test analysis indicated a significant difference in adequate relief groups $(P$-value $=0.014)$, EORTC QLQ-C30 Diarrhea Symptoms domain $(P$-value $<0.001)$, and EORTC GI.NET21 GI Symptoms domain responders and nonresponders $(P$-value $=0.005)$.

A large ES was observed among ISP patients who reported change of "A great deal better" and "Much better" in BMs $(-1.97,-1.83$, respectively), patients who improved in the collapsed perception categories $(-0.99)$, patients who reported being "very satisfied" with the relief $(-1.53)$, improved in the collapsed satisfaction categories $(-1.23)$, and patients who reported their perception of change in BM frequency clinically meaningful $(-1.27)$ (Fig. 2). Kruskal-Wallis Exact test indicated a significant difference between the perceptions of change in $\mathrm{BM}$ response groups $(P$-value $=0.029)$.

\section{Distribution-based thresholds}

Overall, the thresholds for meaningful change in overall average BM frequency at baseline and the change in average $\mathrm{BM}$ frequency from baseline at Week 12 were 0.62 and $0.86 \mathrm{BM} /$ day, respectively (Table 1 ).

\section{Responsiveness}

Responsiveness analysis suggested that change from baseline in overall average BM frequency was moderately responsive to patient reported changes (Table 2). There were large reductions in average weekly BMs among patients indicating a change from "No" at baseline to "Yes" in adequate relief at Week $12(\mathrm{SES}=-0.99)$, patients that indicated a great deal of improvement in BMs $(\mathrm{SES}=-1.44)$, and among patients who were "Very satisfied" (SES $=-1.18)$ with their relief. The number of ISP patients stratified into the perception of change and satisfaction with study medication response was suboptimal.

Responsiveness analysis indicated slightly greater reductions in BM frequency from baseline at Week 12 when compared to change during the Double-blind Treatment Period. Large negative SES values were observed across several item response categories, including patients who changed their response on the adequate relief item to "Yes" at Week 12 as well as those that continued to report "No" (SES $=-1.40$ and -0.81 , respectively), patients whose self-reported perception of change was "A great deal better" (SES = 1.80 ), and among patients who reported being "Very satisfied" with their relief of CS symptoms (SES =1.38).

Cumulative distribution function curves in Figs. 3 and 4 show a clear separation of treatment and placebo at identified thresholds of meaningful change.

Table 1 Summary of Meaningful Change Thresholds

\begin{tabular}{|c|c|c|c|c|c|c|c|c|}
\hline \multirow[t]{3}{*}{ Parameter } & \multirow{3}{*}{$\begin{array}{l}\text { Threshold for } \\
\text { Acceptability }\end{array}$} & \multirow{3}{*}{$\begin{array}{l}\text { Analysis } \\
\text { Population }\end{array}$} & \multicolumn{6}{|c|}{ Average Number of Daily BMs } \\
\hline & & & \multicolumn{3}{|c|}{ Overall Change from Baseline } & \multicolumn{3}{|c|}{ Change from Baseline at Week 12} \\
\hline & & & $\mathrm{N}$ & ES & Mean & $\mathrm{N}$ & ES & Mean \\
\hline \multicolumn{9}{|l|}{ Anchor-based Meaningful Change Thresholds } \\
\hline Adequate relief (changed to Yes at Week 12) & \multirow{3}{*}{$\begin{array}{l}\text { Effect Size: } \\
\text {-Small }(\geq 0.2) \\
\text {-Moderate }(\geq 0.5) \\
\text {-Large }(\geq 0.8)\end{array}$} & ITT & 19 & -1.20 & -1.90 & 18 & -1.58 & -2.52 \\
\hline EORTC QLQ-C30 Diarrhea (Responder) & & $\mathrm{ITT}$ & 45 & -0.83 & -1.71 & 40 & -1.24 & -2.56 \\
\hline EORTC GI.NET21 GI Symptoms (Responder) & & ITT & 30 & -1.25 & -1.88 & 25 & -1.49 & -2.27 \\
\hline \multicolumn{9}{|l|}{ Distribution-based Meaningful Change Thresholds } \\
\hline$-1 / 2$ Baseline SD & N/A & $\mathrm{ITT}$ & 135 & N/A & 0.62 & 108 & N/A & 0.86 \\
\hline
\end{tabular}

BM Bowel movement, EORTC QLQ-C30 European Organization for Research and Treatment of Cancer Quality of Life Questionnaire - Core Questionnaire, ES Effect size, ITT Intent-to-treat, N/A Not applicable, SD Standard deviation 


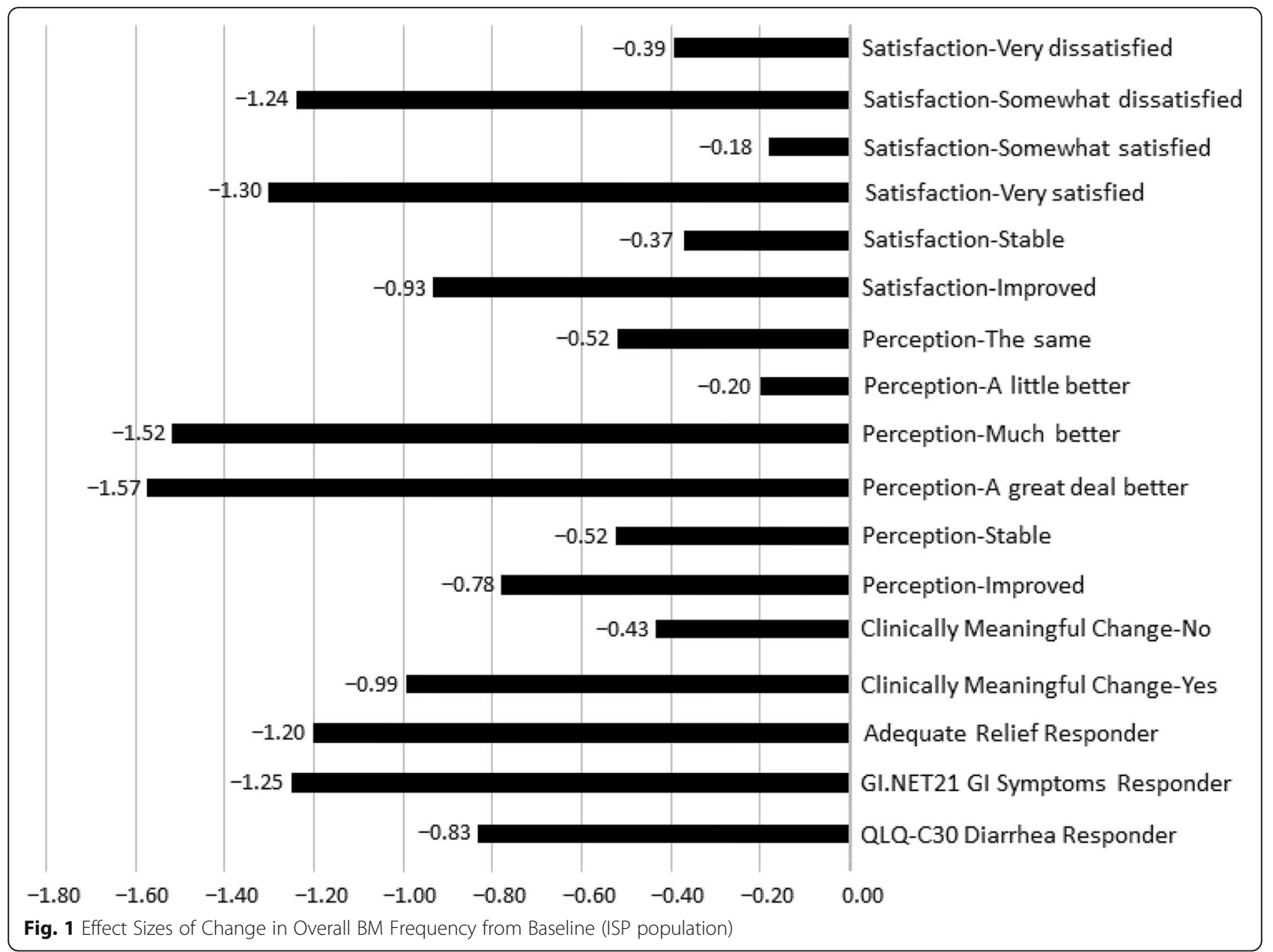

\section{Discussion}

Diarrhea is a prominent feature of CS, which may require a different impact measurement approach than other forms of diarrhea to understand clinically meaningful change. A distribution-based method yielded meaningful change estimates of $0.62 \mathrm{BM} /$ day for overall $\mathrm{BM}$ frequency and $0.83 \mathrm{BM} /$ day at Week 12 , indicating that patients whose average BM frequency increased or decreased by more than that level were reporting a meaningful change. An anchor-based approach was developed using exit interview data and change in scores. The QLQ-C30 Diarrhea Symptom and GI.NET21 GI Symptoms scale were found to have an association $>0.3$, and were thus used to derive estimates of meaningful change. Specifically, change from baseline in the QLQ-C30 Diarrhea symptom score at Week 12 correlated with Change from baseline in Overall BM frequency at 0.38. When applying exit interview data as the basis for meaningful change, patients' perception of change in BMs since beginning the study yielded ES estimates of -1.57 for overall change during the
Double-blind Treatment Period and - 1.97 for change between baseline and Week 12.

When analyzing the responsiveness of BM frequency to various levels of patient reported change, ES estimates for the perception of change item for both overall change and change from Week 12 were significantly different despite the low subject numbers interviewed after the Double-blind Treatment Period. The ES estimates (and the accompanying threshold of $-1.97 \mathrm{BMs}$ ) could be valuable when approximating meaningful change in BM frequency. When applying these thresholds to the clinical change results in the CDF curves, the trend towards greater reductions in BM frequency among the treatment patients indicate a higher percentage would exceed the anchor and distribution-based thresholds, although this would need to be confirmed as part of an efficacy analysis.

Current clinical outcome assessment research has demonstrated that it is possible to establish defensible 


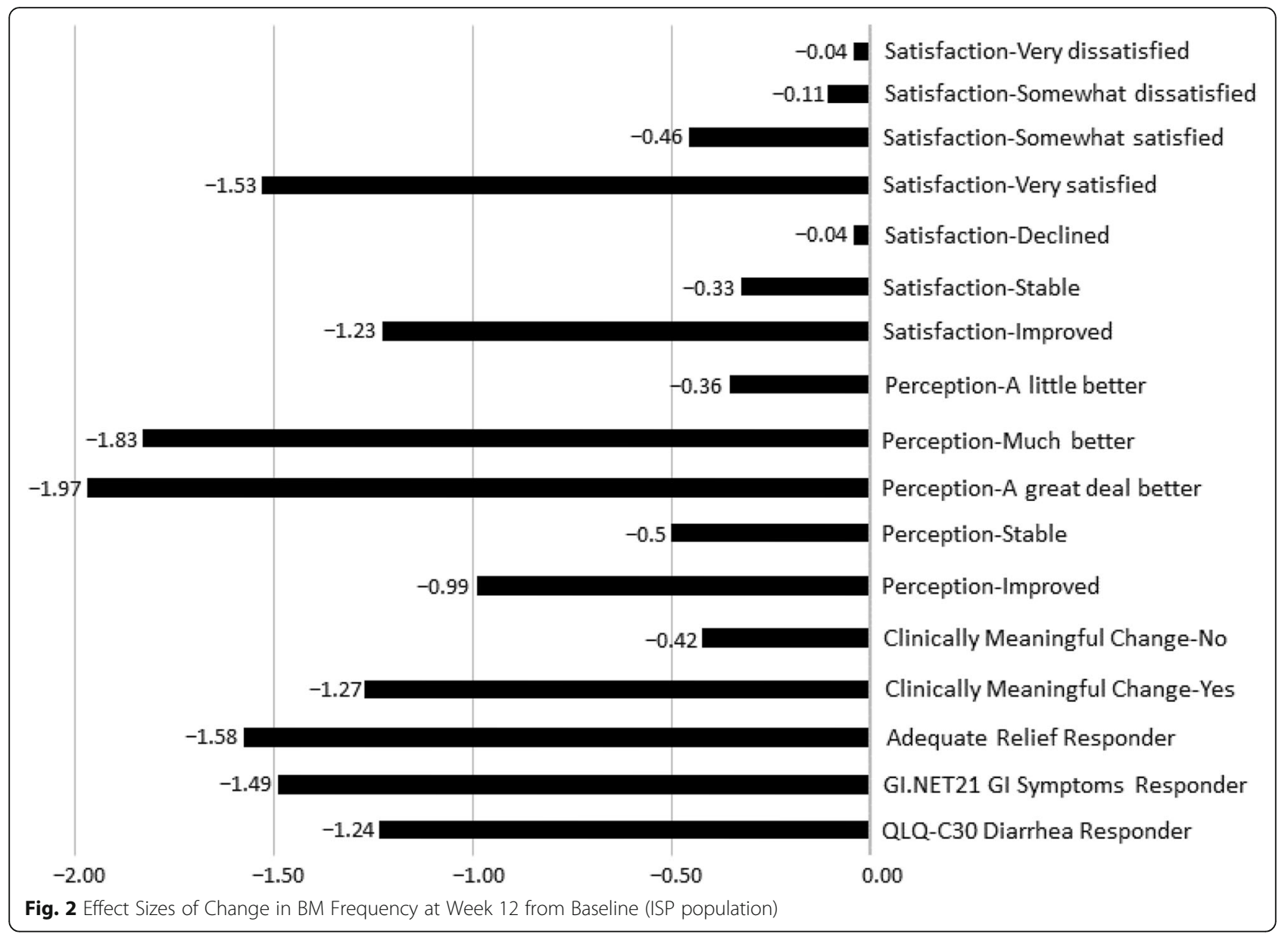

Table 2 Summary of Responsiveness Results

\begin{tabular}{|c|c|c|c|c|c|c|}
\hline \multirow[t]{3}{*}{ Parameter } & \multirow{3}{*}{$\begin{array}{l}\text { Threshold for } \\
\text { Acceptability }\end{array}$} & \multirow{3}{*}{$\begin{array}{l}\text { Analysis } \\
\text { Population }\end{array}$} & \multicolumn{4}{|c|}{ Average Daily BMs } \\
\hline & & & \multicolumn{2}{|c|}{$\begin{array}{l}\text { Overall Change from } \\
\text { Baseline }\end{array}$} & \multicolumn{2}{|c|}{$\begin{array}{l}\text { Change from Baseline at Week } \\
12\end{array}$} \\
\hline & & & $\mathrm{N}$ & SES & N & SES \\
\hline \multicolumn{7}{|l|}{ Responsiveness (SES) } \\
\hline Adequate relief (changed to Yes at Week 12) ${ }^{a}$ & \multirow{4}{*}{$\begin{array}{l}\text { Standard Effect Size: } \\
\text {-Small }(\geq 0.2) \\
\text {-Moderate }(\geq 0.5) \\
\text {-Large }(\geq 0.8)\end{array}$} & ITT & 19 & -0.99 & 18 & -1.40 \\
\hline Perception of change in $\mathrm{BMs}^{\mathrm{b}}$ & & & & & & \\
\hline A great deal better & & ISP & 9 & -1.44 & 8 & -1.80 \\
\hline Much better & & ISP & 4 & -0.50 & 4 & -0.60 \\
\hline \multicolumn{7}{|l|}{ Satisfaction with study medication—Relief of $\mathrm{CS}^{\mathrm{C}}$} \\
\hline Very satisfied & & ISP & 12 & -1.18 & 12 & -1.38 \\
\hline Somewhat satisfied & & ISP & 7 & -0.15 & 6 & -0.38 \\
\hline
\end{tabular}

BM Bowel movement, CS Carcinoid syndrome, ISP Interview subpopulation, ITT Intent-to-treat, SES Standardized effect size

"There were $n=75$ "not changed to yes at Week 12 "

'There were $n=10$ "a little better"; $\mathrm{n}=10$ "the same"; and $\mathrm{n}=1$ "a little worse"

'There were $n=8$ "neither satisfied nor dissatisfied"; $n=3$ "somewhat dissatisfied"; and $\mathrm{n}=3$ "very satisfied" 


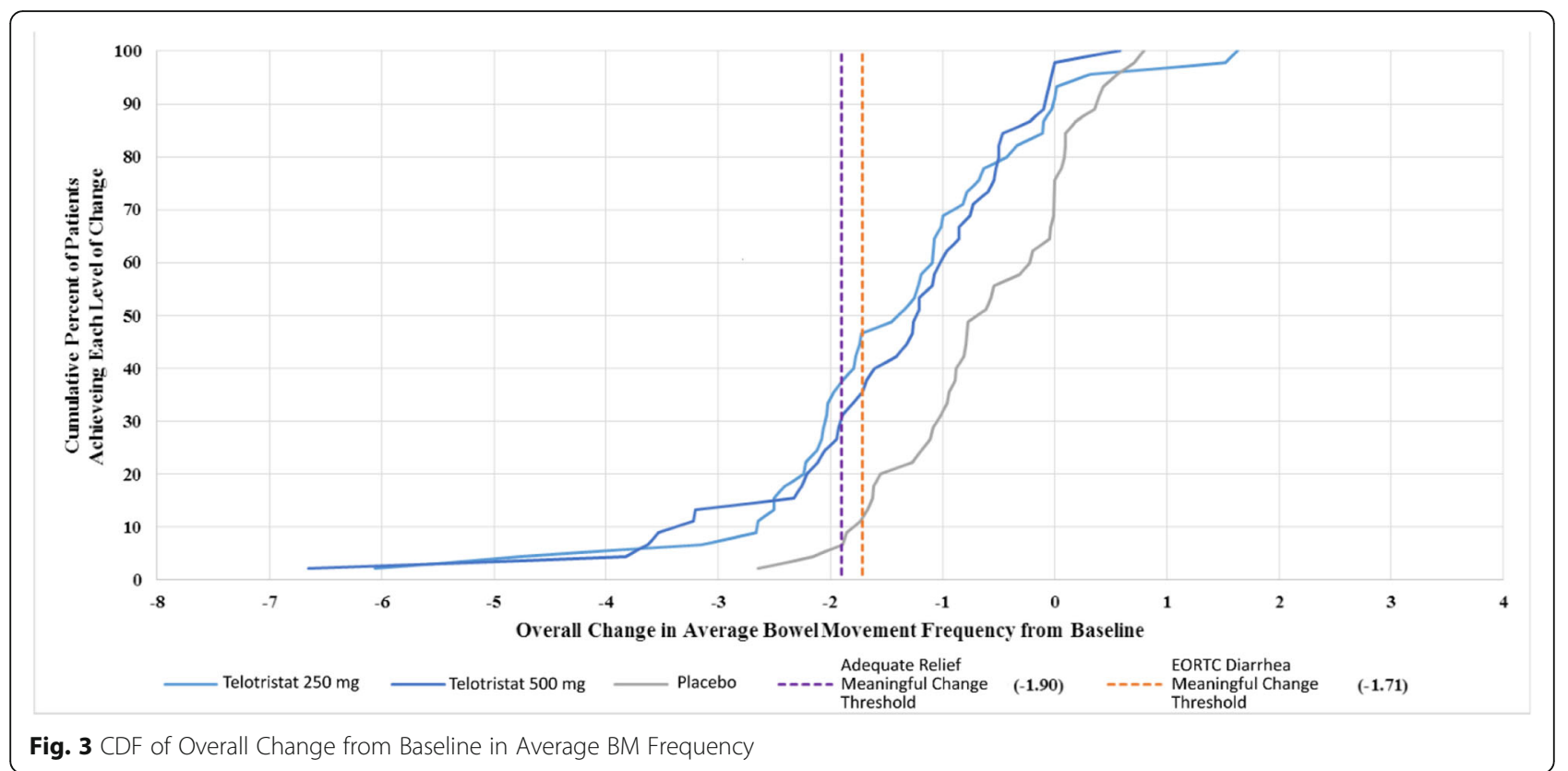

responder thresholds, which support informed treatment decisions [22]. Some physicians interpret clinically meaningful change based on means: however, it is important to examine the distribution of response as in the CDF. The relevance of the CDF is reflected by its inclusion in product labeling [23]. Figure 4 shows that some responders achieved reductions in the range of 3 to $9 \mathrm{BMs} /$ day on treatment, but not on placebo. These are results averaged over 12-weeks, corresponding to $\mathrm{BM}$ reductions during this time frame.
A limitation is that a minimum change is difficult to identify. The mean BM frequency change in a responder group is not necessarily a minimum; it would be of interest to examine empiric density distributions to see which levels of BM frequency change distinguish between responders and non-responders.

\section{Conclusions}

Meaningful change derivation is critical for the interpretation of patient outcomes in the evaluation of treatment efficacy. Analyses identified a meaningful change

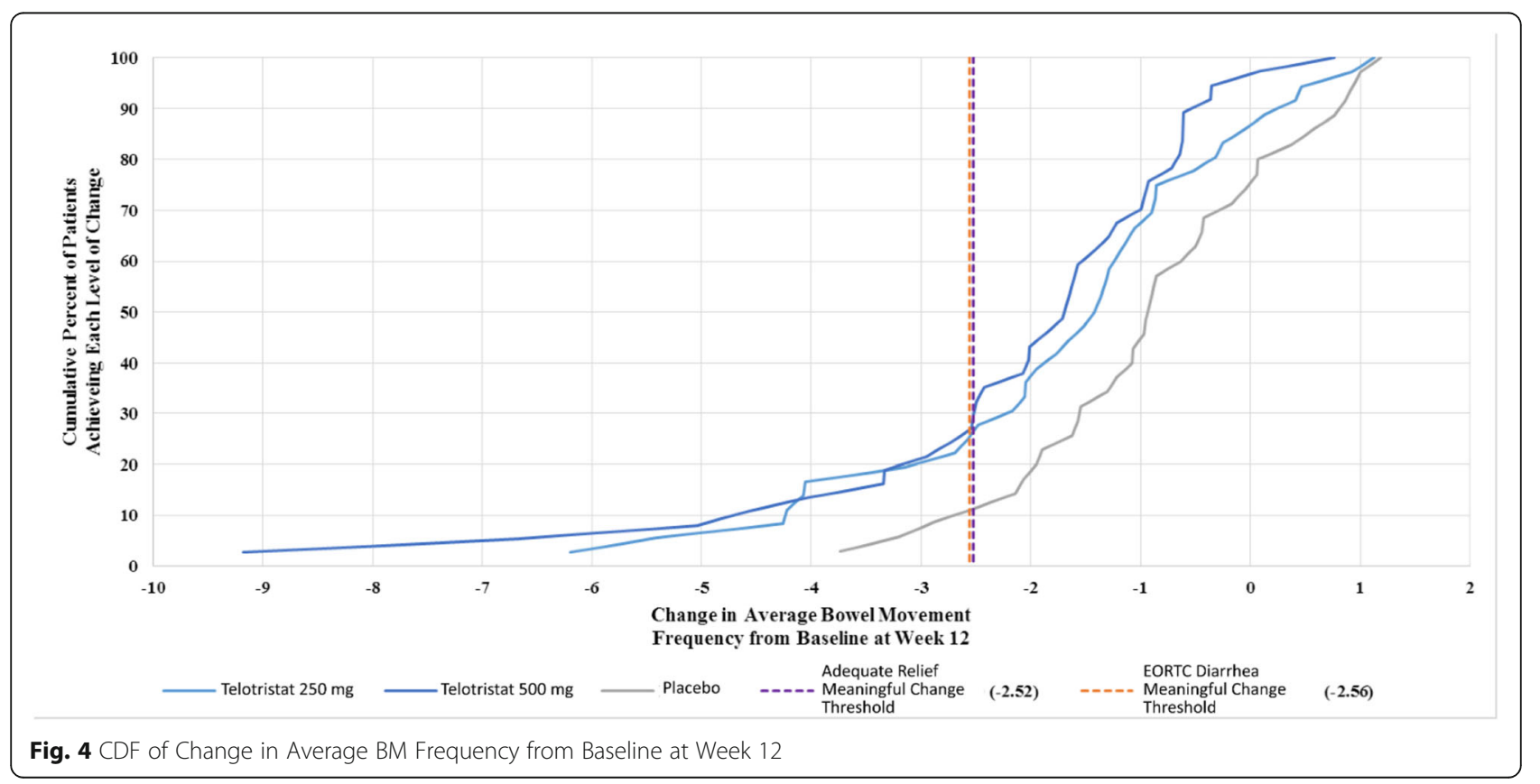


in BM frequency which would coincide with improvement across each of the patient reported anchors (retrospective and prospective). More specifically, CS patients treated with telotristat ethyl experienced clinically meaningful reductions in BM frequency of $\geq 30 \%$ over 12 weeks.

\begin{abstract}
Abbreviations
ANCOVA: Analysis Of Covariance; BM: Bowel movement; CDF: Cumulative distribution function; CS: Carcinoid syndrome; EORTC: European Organization for Research and Treatment of Cancer; ES: Effect size; GI.NET21: EORTC Gastrointestinal NET; HRQoL: Health-related quality of life; ISP: Interview subpopulation; ITT: Intent-to-treat; NET: Neuroendocrine tumors; PRO: Patient-reported outcome; QLQ-C30: Quality of Life Questionnaire Core Questionnaire; SD: Standard deviation; SES: Standardized effect size; SSA: Somatostatin analogs
\end{abstract}

\section{Acknowledgments}

The authors thank the patients who shared their experiences from the TELESTAR clinical trial with the interviewers.

\section{Authors' contributions}

SH: study concepts; study design; data acquisition; quality control of data and algorithms; data analysis and interpretation; statistical analysis; manuscript preparation; manuscript editing; manuscript review. JR: data analysis and interpretation; manuscript preparation; manuscript editing; manuscript review. MK: data analysis and interpretation; manuscript preparation; manuscript editing; manuscript review. EB: data analysis and interpretation; manuscript preparation; manuscript editing; manuscript review. LA: data analysis and interpretation; manuscript preparation; manuscript editing; manuscript review. MC: data analysis and interpretation; manuscript preparation; manuscript editing; manuscript review. KÖ: data analysis and interpretation; manuscript preparation; manuscript editing; manuscript review. MP: data analysis and interpretation; manuscript preparation; manuscript editing; manuscript review. JG: data acquisition; quality control of data and algorithms; data analysis and interpretation; manuscript preparation; manuscript editing; manuscript review. PB: study concepts; study design; data acquisition; data analysis and interpretation; manuscript preparation; manuscript editing; manuscript review. QMY: study concepts; study design; data acquisition; data analysis and interpretation; manuscript preparation; manuscript editing; manuscript review. PL: study concepts; study design; data acquisition; data analysis and interpretation; manuscript preparation; manuscript editing; manuscript review. All authors read and approved the final manuscript.

\section{Funding}

This work was supported by Lexicon Pharmaceuticals, Inc.

\section{Availability of data and materials}

The datasets generated and/or analyzed during the current study are not publicly available due to confidentiality but are available from the corresponding author on reasonable request.

\section{Ethics approval and consent to participate}

This observational study included no administration of any therapeutic or prophylactic agent. There were no anticipated physical risks or direct benefits to patients participating in the study, although patients may become uncomfortable or emotional discussing their experiences with carcinoid syndrome symptoms. As such, participation was completely voluntary and participants could withdraw at any time and still receive (as applicable) the full exit study incentive. Participation in the exit interview was not a requirement for TELESTAR clinical trial participation (e.g., should a patient decide not to participate in an exit interview, they could still continue in any remaining clinical trial activities [e.g., open-label treatment period]). An incentive for participation in the exit interview substudy was provided, if approved by each participating clinical site's local ethics review board, to compensate patients for their time. The amount and payment methods was reviewed and approved by each ethics committee to ensure that payments (if allowed) were commensurate with the time needed to participate in the interview and were not coercive.
Consent for publication

Not applicable.

\section{Competing interests}

S. Hudgens and J. Gable are employed by Clinical Outcomes Solutions, the organization contracted by Lexicon to analyze the data and develop this manuscript. These authors have no additional financial relationships or otherwise to declare. M. Kulke has received consulting fees from Lexicon, Novartis, and Ipsen outside the submitted work. E. Bergsland is an uncompensated adviser for Lexicon Pharmaceuticals, Inc., and Ipsen. L. Anthony has received grants from Lexicon Pharmaceuticals Inc. M. Caplin has received compensation from Lexicon Pharmaceuticals Inc for participation on an advisory board. K. Öberg has received honoraria and speakers' bureau fees from Novartis and Ipsen. M. Pavel has received consulting fees from Lexicon Pharmaceuticals Inc, Novartis, and Ipsen. P. Banks, Q.M. Yang, and P. Lapuerta are all currently employees of Lexicon Pharmaceuticals Inc and own stock. The authors have declared that they have no other competing interests regarding the content of this article.

\section{Author details}

${ }^{1}$ CEO \& Strategic Lead, Quantitative Science, Clinical Outcomes Solutions, 1790 E. River Rd, Suite 205, Tucson, AZ 85718, USA. ${ }^{2}$ Hampshire Hospitals NHS Foundation Trust, Aldermaston Road, Basingstoke, Hampshire RG24 9NA, UK. ${ }^{3}$ Dana-Farber Cancer Institute, 450 Brookline Ave, Boston, MA 02215, USA. ${ }^{4}$ UCSF Helen Diller Family Comprehensive Cancer Center, 1450 3rd St, San Francisco, CA 94158, USA. ${ }^{5}$ University of Kentucky, 410 Administration Dr, Lexington, KY 40508, USA. ${ }^{6}$ Royal Free Hospital, Pond St, Hampstead, London NW3 2QG, UK. 'Uppsala University, 75236 Uppsala, Sweden. ${ }^{8}$ Friedrich Alexander University Erlangen-Nürnberg, Schloßplatz 4, 91054 Erlangen, Germany. ' ${ }^{2}$ exicon Pharmaceuticals Inc., 8800 Technology Forest PI, The Woodlands, TX, USA.

Received: 18 March 2019 Accepted: 25 September 2019

Published online: 26 October 2019

\section{References}

1. Robertson, R., Geiger, W., \& Davis, N. (2006). Carcinoid tumors. American Family Physician, 74, 429-434.

2. Beaumont, J., Cella, D., Phan, A., Choi, S., Liu, Z., \& Yao, J. (2012). Comparison of health-related quality of life in patients with neuroendocrine tumors with quality of life in the general US population. Pancreas, 41, 461-466.

3. Kaupp-Roberts, S., Srirajaskanthan, R., \& Ramage, J. (2015). Symptoms and quality of life in gastroenteropancreatic neuroendocrine tumours. EMJ Oncology, 3, 34-40.

4. Ramage, J., \& Davies, A. (2003). Measurement of quality of life in carcinoid/ neuroendocrine tumours. Endocrine-Related Cancer, 10, 483-486.

5. Yadegarfar, G., Friend, L., Jones, L., Plum, L., Ardill, J., Taal, B., Larsson, G. Jeziorski, K., Kwekkeboom, D., \& Ramage, J. (2013). Validation of the EORTC QLQ-GINET21 questionnaire for assessing quality of life of patients with gastrointestinal neuroendocrine tumours. British Journal of Cancer, 108, 301-310.

6. Modlin, I., Lye, K., \& Kidd, M. (2003). A 5-decade analysis of 13,715 carcinoid tumors. Cancer, 97, 934-959.

7. Zuetenhorst, J., \& Taal, B. (2005). Metastatic carcinoid tumors: A clinical review. Oncologist, 10, 123-131.

8. (2017) National Cancer Institute. Gastrointestinal carcinoid tumors treatment (PDQ) - health professional version. Available at: https://www. cancer.gov/types/gi-carcinoid-tumors/hp/gi-carcinoid-treatment-pdq. Accessed 15 June 2018.

9. Bhattacharyya, S., Toumpaakis, C., Chilkunda, D., Caplin, M., \& Davar, J. (2011). Risk factors for the development and progression of carcinoid heart disease. The American Journal of Cardiology, 107, 1221-1226.

10. Moller, J., Connolly, H., Rubin, J., Seward, J., Modesto, K., \& Pellikka, P. (2003). Factors associated with progression of carcinoid heart disease. The New England Journal of Medicine, 348, 1005-1015.

11. Druce, M., Rockall, A., \& Grossman, A. (2009). Fibrosis and carcinoid syndrome: From causation to future therapy. Nature Reviews. Endocrinology, $5,276-283$

12. (2017) National Institutes of Health. ClinicalTrials.gov website. Available at: https://clinicaltrials.gov/ct2/show/NCT01677910. Accessed 15 June 2018. 
13. Kulke, M., Horsch, D., Caplin, M., Anthony, L., Bergsland, E., Oberg, K., Welin, S., Warner, R., Lombard-Bohas, C., Kunz, P., Grande, E., Valle, J., Fleming, D., Lapuerta, P., Banks, P., Jackson, S., Zambrowicz, B., Sands, A., \& Pavel, M. (2017). Telotristat ethyl, a tryptophan hydroxylase inhibitor for the treatment of carcinoid syndrome. Journal of Clinical Oncology, 35, 14-23.

14. Aaronson, N., Ahmedzai, S., Bergman, B., Bullinger, M., Cull, A., Duez, N., Filiberti, A., Flechtner, H., Fleishman, S., \& de Haes, J. (1993). The European Organization for Research and Treatment of cancer QLQ-C30: A quality-oflife instrument for use in international clinical trials in oncology. Journal of the National Cancer Institute, 85, 365-376.

15. (2017) EORTC Quality of Life. EORTC QLQ-GI.NET21. Available at: http:// groups.eortc.be/qol/sites/default/files/img/slider/specimen_gi.net21_english. pdf. Accessed 15 June 2018.

16. Anthony, L., Ervin, C., Lapuerta, P., Kulke, M., Kunz, P., Bergsland, E., Horsch, D. , Metz, D., Pasieka, J., Pavlakis, N., Pavel, M., Caplin, M., Oberg, K., Ramage, J., Evans, E., Yang, Q., Jackson, S., Arnold, K., Law, L., \& DiBenedetti, D. (2017). Understanding the patient experience with carcinoid syndrome: Exit interviews from a randomized, placebo-controlled study of telotristat ethyl. Clinical Therapeutics, (17), 30987-30986. https://doi.org/10.1016/j.clinthera. 2017.09.013 [Epub ahead of print].

17. (2013). SAS version 9 [computer software]. Cary: SAS Institute Inc.

18. Revicki, D., Hays, R., Cella, D., \& Sloan, J. (2008). Recommended methods for determining responsiveness and minimally important differences for patient-reported outcomes. Journal of Clinical Epidemiology, 61, 102-109.

19. Litwin, M. S. (1995). How to measure survey reliability and validity. Thousand Oaks: Sage Publications.

20. Kazis, L., Anderson, J., \& Meenan, R. (1989). Effect sizes for interpreting changes in health status. Medical Care, 27, S178-S189.

21. Cohen J (1977) Statistical power analysis for the behavioral sciences (rev. Lawrence Erlbaum Associates, Inc).

22. Coon, C., \& Cook, K. (2017). Moving from significance to real-world meaning: Methods for interpreting change in clinical outcome assessment scores. Quality of Life Research. https://doi.org/10.1007/s11136-017-1616-3 [Epub ahead of print].

23. (2017) Xermelo (telotristat ethyl) prescribing information. United States Food and Drug Administration. Available at: https://www.accessdata.fda. gov/drugsatfda_docs/label/2017/208794s00olbl.pdf. Accessed 10 July 2018

\section{Publisher's Note}

Springer Nature remains neutral with regard to jurisdictional claims in published maps and institutional affiliations.

\section{Submit your manuscript to a SpringerOpen ${ }^{\circ}$ journal and benefit from:}

- Convenient online submission

- Rigorous peer review

- Open access: articles freely available online

- High visibility within the field

- Retaining the copyright to your article

Submit your next manuscript at $\boldsymbol{\nabla}$ springeropen.com 